Methods We used immunohistochemistry (IHC) and immunofluorescent (IF) co-staining approaches involving cell-specific markers $\alpha$ SMA (myofibroblast), CK7 (epithelium) and CD68 (infiltrating cells e.g., macrophages) in formalin fixed paraffin embedded (FFPE) lung tissues from IPF patients $(n=3)$.

Results Using both immunoperoxidase and immunofluorescence co-staining, we have demonstrated PKM2 expression in both the CK7 positive bronchial epithelium, as well as the CK7 positive epithelium overlying fibrotic foci in lungs from IPF patients. PKM2 was also co-localised with CD68 positive infiltrating macrophage populations. Occasional examples of PKM2 positive staining were observed in $\alpha$ SMA-positive myofibroblasts.

Conclusions PKM2 expression was observed in epithelium and infiltrating macrophages, thought to be critically involved in the pathology of IPF. These data suggest that these cells may rely on aerobic glycolysis to provide for their biosynthetic requirements, and provide a foundation for studies to investigate the role of glycolytic reprogramming in lung injury and fibrosis.

\section{P51 LUNG EPITHELIAL CELL INHIBITION OF CYTOKINE PRODUCTION BY PERIPHERAL BLOOD MONONUCLEAR CELLS AND LUNG LYMPHOCYTES}

S Layzell, A Southern, ID Pavord, TJ Powell. Respiratory Medicine Unit and NIHR Oxford Biomedical Research Centre Respiratory Theme, Nuffield Department of Clinical Medicine, University of Oxford, Oxford, UK

\subsection{6/thoraxjnl-2017-210983.193}

Introduction Type 2 cytokines such as IL-5, IL-13 and IL-4 produced by primed type $2 \mathrm{~T}$ cells have been shown to be important in the pathogenesis of eosinophilic airway inflammation. Factors regulating the state of activation of these cells are incompletely understood. We and others have shown that release of IL-13 by stimulated T cells can be inhibited by epithelial cells. This study used a PBMC based bulk culture system to: 1) determine whether production of other type-2 cytokines is inhibited by co-culture with epithelial cells; 2) compare inhibition of activated PBMC and human lung lymphocytes and 3) investigate whether specific soluble mediators modified inhibition of IL-13 release. PBMC isolated from blood and lymphocytes isolated from lung tissue were cultured with IL-2 for five days in the absence and presence of A549 and BEAS2B epithelial cells. The cytokines IL-13, IL-5, IL-9 and $\mathrm{TNFa}$ were measured in the supernatant of these cells. Similar co-culture experiments were performed in the presence of different inhibitors or blocking cytokine antibodies.

Results Production of all cytokines measured were reduced in the presence of epithelial cells: IL-13 shown as mean $\mathrm{pg} / 10^{6}$ cells+/-SD in $200 \mathrm{U} / \mathrm{ml}$ IL-2) PBMC: 1184+/-24,+A549 12 $+/-1$ or lung cells $795+/-158,+$ A549 $50+/-2$. PBMC and lung cells were inhibited to a similar degree although, importantly, lung cells produced more IL-9 and less TNFa than a comparable number of PBMC. We found that adding inhibitors to IL10, TGF- $\beta$, Aryl Hydrocarbon Receptor (AHR blocked with $\mathrm{CH}-223191$ ), prostaglandins (indomethacin) and nitric oxide (NMMA) did not alter the A549 mediated regulation of IL-13 release by the PBMC or lung cells.

Conclusions The inhibition of cytokine release by PBMC and lung cells in the presence of epithelial cells could indicate generalised regulation of inflammatory cytokine release. Blocking of IL-10, TGF- $\beta$, AHR, prostaglandins and nitric oxide was not able to reduce the regulation of the cytokine release but more specific inhibitors or further titration may be required. The characterisation of cells from lung tissue and the regulation of these by epithelial cells could further elucidate possible ways of regulating and reducing cellular inflammatory responses in asthma.

\section{P52 EXPLORING THE INTERACTION BETWEEN HIV-1 GP120, BRONCHIAL AIRWAY EPITHELIAL CELLS AND MACROPHAGES}

B Talbot, CA Stokes, PJ Collini, AM Condliffe. Department of Infection, Immunity and Cardiovascular Disease, University of Sheffield, Sheffield, UK

\subsection{6/thoraxjnl-2017-210983.194}

Rationale and Hypothesis HIV-1-seropositive individuals receiving highly active antiretroviral therapy (HAART) have an increased incidence of chronic obstructive pulmonary disease (COPD), independent of smoking history. Although HIV-1 infection is associated with impaired redox homeostasis and increased pro-inflammatory cytokine expression in the lungs despite HAART, the mechanisms driving HIV-1-associated COPD are poorly understood. Free HIV-1 envelope glycoprotein gp120 is detectable in bronchoalveolar lavage fluid from HAART-treated individuals. gp120 displays affinity (tropism) for either CCR5 or CXCR4 chemokine receptors, and has been implicated as a mediator of inflammation and oxidative stress in various HIV-1-associated disease processes. We hypothesised that gp120 directly induces bronchial epithelial cell oxidative stress, and drives airway inflammation indirectly via alveolar macrophages, a response which is augmented following secondary exposure to pro-inflammatory stimuli such as bacterial pathogens.

Objectives To explore the mechanisms and consequences of gp120 interactions with bronchial epithelial cells and macrophages.

Methods An immortalised bronchial epithelial cell line (BEAS2B), primary bronchial epithelial cells (PBECs) or monocytederived macrophages (MDMs) from healthy volunteers were treated with recombinant gp120 (CCR5- or CXCR4-tropic, $100 \mathrm{ng} / \mathrm{mL}$ ) for 24-48 hour. BEAS-2B were primed (or not) with IL-1B. MDMs were co-cultured with confluent BEAS-2B cells at a ratio of $1: 5$ in the presence or absence of LPS $(100 \mathrm{ng} / \mathrm{ml})$. Cytokine outputs were quantified by ELISA, and cellular reactive oxygen species (ROS) production assessed by confocal microscopy using CellROX or MitoSOX reagents.

Findings Picomolar concentrations of CXCR4- but not CCR5tropic gp120 induced CXCL8 release from IL-1ß-primed BEAS-2B monocultures and upregulated cellular ROS production in both BEAS-2Bs and PBECs, consistent with expression of CXCR4 but not CCR5 on these cells. gp120 stimulation of BEAS-2B/MDM co-cultures caused no detectable changes in cytokine release. However, co-cultures primed with gp120 (of either tropism) and stimulated with LPS demonstrated significant CXCL8 release at 48 hours, reflecting MDM expression of both chemokine receptors. Impaired redox homeostasis and upregulated inflammatory responses may contribute to gp120mediated airway epithelial dysfunction, and may drive neutrophil recruitment in this setting. 